\title{
NUMERICAL APPLICATIONS FOR INSULIN TREATMENT MODELS
}

\author{
BY \\ VIOREL ARNĂUTU and ANA-MARIA MOŞNEAGU
}

\begin{abstract}
We investigate two models for dynamics of the insulin and glucose concentration for diabetic human patients. The first model is a minimal one with two compartments and the second one is obtained by introducing a reaction term. The blood glucose control problem has been seen as an optimal control problem in order to obtain a scheme of insulin treatment. We present corresponding numerical experiments and we compare the two models.
\end{abstract}

Mathematics Subject Classification 2000: 49N25, 65K10, 92C50.

Key words: insulin-glucose system, diabetes of type I, optimal control problem.

\section{Introduction}

We consider models for insulin treatment for patients with diabetes. The main problem is to keep the blood glucose level close to a convenient value and to avoid large variations of it. In the case of diabetes, the pancreas is not able to provide insulin enough for the metabolism of glucose. Blood glucose concentration increases when glucose is administrated while insulin accelerates the removal of glucose from the plasma. Therefore the blood sugar decays to a normal value of $0.8-1.2 \mathrm{~g} / \mathrm{l}$. We consider diabetic human patients who are not able to produce insulin enough. The insulin is supplied by injections. The glucose concentration can be easily measured. A detailed model for the dynamics of the insulin-glucose system is presented in [7]. An overview on mathematical models for the insulin-glucose system is presented in [12] and [13]. We also cite the monograph [11].

We study two models for dynamics of the insulin and glucose concentration. The first model, a simplified one with two compartments was in- 
troduced in [5] and studied in [10] and also in [6, Chapter 6.2]. A reaction term is added to obtain the second model. For details about reaction terms we send to [1] and [2]. We consider two distinct optimal control problems(corresponding to the models above) to obtain a schedule of insulin treatment providing a good control of glycemia over some time interval. The control considered is of impulsive type. We obtain first order necessary optimality conditions which are used for optimization algorithms. Numerical tests are done. The numerical tests show that the first model works well only for initial cocentrations of insulin and glucose between appropriate medical limits. For initial cocentrations of insulin and glucose outside the usual medical limits it is possible to obtain negative or not allowed values for the concentration of insulin injected and for the concentration of glucose and therefore the model fails. The second model works well even in this situation.

For applied numerical methods (finite differences) see, for instance, [8].

\section{A model for insulin-glucose dynamics}

Let us denote by $I(t)$ the concentration of insulin injected, and by $G(t)$ the concentration of glucose at the moment $t \in[0, L]$, where $L>0$ is the length of the time interval considered to study the evolution of insulin and glucose.

We denote by $I_{0}$ the initial concentration of insulin and by $G_{0}$ the initial concentration of glucose. We consider that $|d|$, where $d<0$, is the decay rate of insulin, $a$ is the growth rate of glucose $(a \neq d)$, and $b$ is a negative constant that can be measured. A corresponding simplified model for dynamics of insulin-glucose system is the following one:

$$
\begin{cases}I^{\prime}(t)=d I(t), & t \in(0, L) \\ G^{\prime}(t)=b I(t)+a G(t), & t \in(0, L) \\ I(0)=I_{0}, G(0)=G_{0} . & \end{cases}
$$

For a normal organism a minimal model with two compartments was introduces in [5] and studied in [10]; see also [6, Chapter 6.2].

System (1) can be integrated mathematically. The solution of (1) is given by the following formulae:

$$
\begin{cases}I(t)=I_{0} e^{d t}, & t \in[0, L] \\ G(t)=G_{0} e^{a t}+\frac{b I_{0}}{d-a}\left(e^{d t}-e^{a t}\right), & t \in[0, L] .\end{cases}
$$


The blood glucose control problem has been considered as an optimal control problem in [9] and [14].

We consider now an optimal control problem with impulsive control to obtain a scheme of insulin treatment to control the blood sugar over some time interval. We denote by $A$ the desired level of glucose. We assume that the initial concentration of insulin is $I(0)=0$ and the patient gets $m$ injections of insulin at the following discrete moments of time: $0=$ $t_{1}<t_{2}<\ldots<t_{m}=L$, with corresponding quantities of insulin $c_{j}=c\left(t_{j}\right)$, $j=1,2, \ldots m$. We denote by $h=t_{j+1}-t_{j}, j=1,2, \ldots, m$, which means time equidistant injections. The dynamics of insulin-glucose system is described by

$$
\left\{\begin{array}{l}
I^{\prime}(t)=d I(t)+\sum_{j=1}^{m} c_{j} \delta_{t_{j}}, \\
G^{\prime}(t)=b I(t)+a G(t), \quad t \in(0, L) \\
I(0)=0, G(0)=G_{0},
\end{array}\right.
$$

where $\delta_{t_{j}}$ is the Dirac mass at $t_{j}$. The solution of (3) in the sense of the theory of distributions is

(4) $\left\{\begin{array}{l}I(t)=\sum_{j=1}^{m} c_{j} H\left(t-t_{j}\right) e^{d\left(t-t_{j}\right)}, \\ G(t)=G_{0} e^{a t}+\frac{b}{d-a} \sum_{j=1}^{m} c_{j} H\left(t-t_{j}\right)\left[e^{d\left(t-t_{j}\right)}-e^{a\left(t-t_{j}\right)}\right], \quad t \in[0, L]\end{array}\right.$

where $H$ is the Heaviside function, i.e., $H: \mathbb{R} \rightarrow \mathbb{R}$ is defined as

$$
H(t)= \begin{cases}1, & \text { if } t \geq 0 \\ 0, & \text { if } t<0\end{cases}
$$

Therefore, the function $t \mapsto H\left(t-t_{j}\right)$ from formulae (4) is defined for $t \in[0, L]$ by

$$
H\left(t-t_{j}\right)= \begin{cases}1, & \text { if } t \in\left[t_{j}, L\right] \\ 0, & \text { if } t \in\left[0, t_{j}\right)\end{cases}
$$

The optimal control problem related to (3) is:

$$
\text { Minimize } \Psi(c)=\frac{1}{2} \int_{0}^{L}[G(t)-A]^{2} d t
$$


subject to $c=\left(c_{1}, c_{2}, \ldots, c_{m}\right) \in \mathbb{R}^{m}$, where $(I, G)$ is the solution of (3). The vector $c$ is the control, an impulsive one - a control that acts only at some discrete moments of time.

Since the functional $\Psi$ is quadratic with respect to every $c_{j}$, it means that there exists at least an optimal control $c=\left(c_{1}, c_{2}, \ldots, c_{m}\right) \in \mathbb{R}^{m}$. The optimal control satisfies:

$$
\frac{\partial \Psi}{\partial c_{j}}(c)=0, j=1,2, \ldots, m,
$$

a linear algebraic system with the unknowns $c_{j}, j=1,2, \ldots, m$. We calculate the partial derivatives and using the formulae (6) we obtain the following algebraic linear system

$$
\sum_{i=1}^{m} q_{i j} c_{i}=B_{j}, j=1,2, \ldots, m,
$$

where

$$
q_{i j}=\frac{b}{d-a} \int_{0}^{L} H\left(t-t_{i}\right) H\left(t-t_{j}\right) e_{i}(t) e_{j}(t) d t
$$

$$
B_{j}=\int_{t_{j}}^{L} e_{j}(t)\left(A-G_{0} e^{a t}\right) d t
$$

with $i, j=1,2, \ldots, m$ and $e_{j}(t)=e^{d\left(t-t_{j}\right)}-e^{a\left(t-t_{j}\right)}, t \in[0, L], j=1,2, \ldots, m$.

If $i>j$, then $t_{i}>t_{j}$ and from (8) we get

$$
q_{i j}=\frac{b}{d-a} \int_{t_{i}}^{L} e_{i}(t) e_{j}(t) d t .
$$

We must calculate the transpose of the matrix $Q=\left[q_{i j}\right]$. Also we need to calculate $B=\left(B_{j}\right)$. We can thus solve system (7) and obtain the optimal control $c \in \mathbb{R}^{m}$. The algorithm is

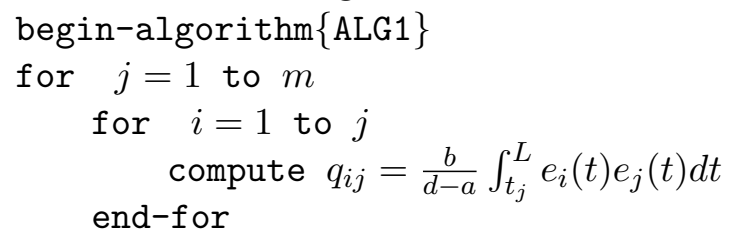




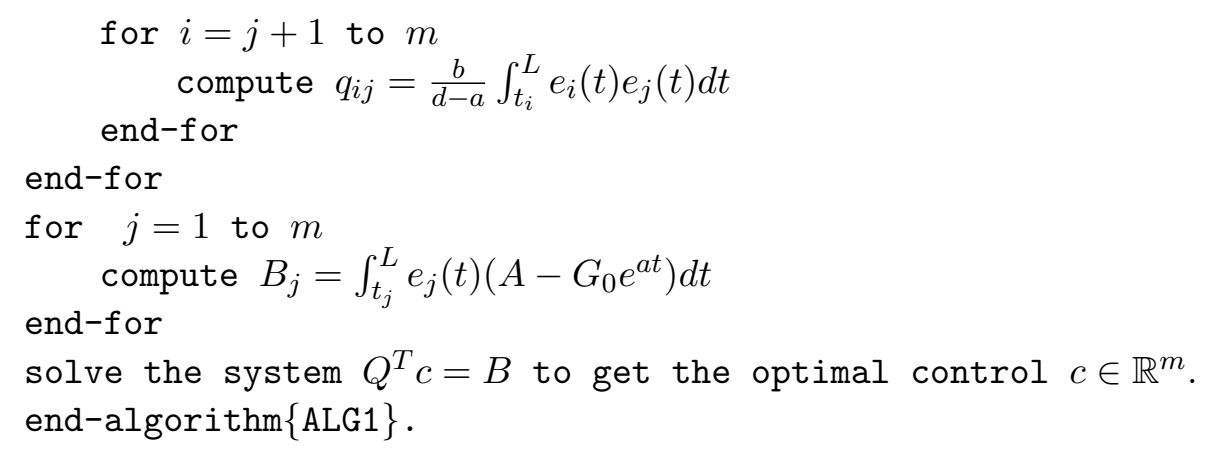

\section{A second model for insulin-glucose dynamics}

We investigate another model for the dynamics of insulin-glucose system, namely:

$$
\begin{cases}I^{\prime}(t)=d I(t), & t \in(0, L) \\ G^{\prime}(t)=b I(t) G(t)+a G(t), & t \in(0, L) \\ I(0)=I_{0}, G(0)=G_{0}, & \end{cases}
$$

where $a, b, d, I(t), G(t), I_{0}, G_{0}$ have the same meanings as in the model (1). Let us remark that the term $b I(t)$ from the second equation in (1) is replaced by the reaction term $b I(t) G(t)$. For details about reaction terms we send to [1] and [2].

This system can be solved mathematically. We consider the problem of insulin dynamics

$$
\left\{\begin{array}{l}
I^{\prime}(t)=d I(t), \quad t \in(0, L) \\
I(0)=I_{0}
\end{array}\right.
$$

which has a unique solution

$$
I(t)=I_{0} e^{d t}, t \in[0, L] .
$$

We use the form of $I(t)$ previously determined and we obtain the following model for the glucose dynamics

$$
\left\{\begin{array}{l}
G^{\prime}(t)=b I_{0} e^{d t} G(t)+a G(t), \quad t \in(0, L) \\
G(0)=G_{0}
\end{array}\right.
$$


which gives the formula for the glucose concentration

$$
G(t)=G_{0} e^{a t} e^{\frac{b}{d} I_{0}\left(e^{d t}-1\right)}, t \in[0, L] .
$$

We now consider the optimal control problem

$$
\text { Minimize } \Psi(c)=\frac{1}{2} \int_{0}^{L}[G(t)-A]^{2} d t
$$

subject to $c=\left(c_{1}, c_{2}, \ldots, c_{m}\right) \in \mathbb{R}^{m}$, where $(I, G)$ is the solution of the model:

$$
\left\{\begin{array}{l}
I^{\prime}(t)=d I(t)+\sum_{j=1}^{m} c_{j} \delta_{t_{j}} \\
G^{\prime}(t)=b I(t) G(t)+a G(t), \quad t \in(0, L) \\
I(0)=0, G(0)=G_{0}
\end{array}\right.
$$

We investigate the optimal control problem under the control constraints $c_{j} \geq 0, j=1,2, \ldots, m$.

The solution of system (17) in the sense of the distributions is

$$
\left\{\begin{array}{l}
I(t)=\sum_{j=1}^{m} c_{j} H\left(t-t_{j}\right) e^{d\left(t-t_{j}\right)}, \\
G(t)=G_{0} e^{a t} e^{\frac{b}{d} \sum_{j=1}^{m} c_{j} H\left(t-t_{j}\right)\left[e^{d\left(t-t_{j}\right)}-1\right]}, \quad t \in[0, L] .
\end{array}\right.
$$

The control $c$ is also an impulsive one. The optimal control (that exists because the functional $\Psi$ is quadratic) satisfies

$$
\nabla \Psi(c)=0,
$$

i.e.,

$$
\frac{\partial \Psi}{\partial c_{j}}(c)=0, j=1,2, \ldots, m
$$

We calculate the partial derivatives and we obtain

$$
\frac{\partial \Psi}{\partial c_{j}}(c)=\int_{0}^{L}[G(t)-A] \frac{\partial G(t)}{\partial c_{j}} d t, j=1,2, \ldots, m,
$$


where

$$
\frac{\partial G(t)}{\partial c_{j}}=G_{0} e^{a t} \frac{b}{d} H\left(t-t_{j}\right)\left[e^{d\left(t-t_{j}\right)}-1\right] e^{\frac{b}{d} \sum_{j=1}^{m} c_{j} H\left(t-t_{j}\right)\left[e^{d\left(t-t_{j}\right)}-1\right]},
$$

Therefore, $\frac{\partial G(t)}{\partial c_{j}}=G(t) \frac{b}{d} H\left(t-t_{j}\right)\left[e^{d\left(t-t_{j}\right)}-1\right], t \in[0, L]$.

We introduce restrictions of the form $0 \leq c_{j} \leq \bar{c}, j=1,2, \ldots, m$, with $\bar{c}$ the maximum amount of insulin injected and we use a projected gradient method to solve the optimal control problem (16).

The conceptual algorithm (Uzawa's algorithm with Armijo method for the steplength $\rho$ ) is:

begin-algorithm $\{$ ALG2 $\}$

S0: $\quad$ set $k=0$

$$
\begin{aligned}
& \text { choose } c^{(0)} \in K=[0, \bar{c}]^{m} \\
& \text { set } \rho^{(0)}=1 \\
& \text { choose } \beta \in(1 / 2 ; 4 / 5)
\end{aligned}
$$

S1: $\quad \rho=\rho^{(k)}$

$$
\begin{aligned}
& \text { S11: } \text { if } \quad \Psi\left(P_{K}\left(c^{(k)}-\rho \nabla \Psi\left(c^{(k)}\right)\right)\right)<\Psi\left(c^{(k)}\right) \\
& \text { then } \quad \rho^{(k+1)}=\rho \\
& \text { else } \quad \rho=\beta \rho \text { go to S11 } \\
& \text { end-if } \quad \\
& \text { S12: } \quad c^{(k+1)}=P_{K}\left(c^{(k)}-\rho^{(k+1)} \nabla \Psi\left(c^{(k)}\right)\right)
\end{aligned}
$$

S2: if $\left\|c^{(k+1)}-c^{(k)}\right\|<\varepsilon$ (the stopping criterion,

$$
\text { where } \varepsilon \text { is a given value) }
$$

then $\operatorname{STOP}\left(c^{(k+1)}\right.$ is the approximating control)

else $k=k+1$

go to $\mathrm{S} 1$

end-if

end-algorithm $\{$ ALG2 $\}$

For details about Uzawa's algorithm and Armijo's rule see [4, Chapter $2.5]$ and [3].

The projection operator $P_{K}: \mathbb{R}^{m} \rightarrow K$ is defined as

$$
P_{K}(w)=\left(P_{[0, \bar{c}]}\left(w_{j}\right)\right)_{j=\overline{1, m}},
$$


where $w=\left(w_{j}\right)_{j=\overline{1, m}}$ and $P_{[0, \bar{c}]}\left(w_{j}\right)= \begin{cases}0, & w_{j}<0 \\ w_{j}, & 0 \leq w_{j} \leq \bar{c} \\ \bar{c}, & w_{j}>\bar{c} .\end{cases}$

\section{Numerical tests}

For both models already described we have indicated algorithms to solve the optimal control problems. For the first model, one has to solve a linear algebraic system whose solution is the optimal control $c \in \mathbb{R}^{m}$ (ALG1). This algorithm has no control constrains. For the second model, to solve the optimal control problem we have used a projected gradient method because of control constrains, namely Uzawa's algorithm with Armijo's rule for the steplength $\rho$. Both algorithms were implemented in Matlab.

For the identification of the values of coefficients $a, b$, and $d$, see $[6$, Chapter 6.2]. For all numerical examples we have considered $a=0.1$, $b=-0.05$, and $d=-0.5$.

Example 1. We take the final time $L=12$ (hours), the initial concentration of glucose $G_{0}=2.2$, the desired level of glucose $A=0.9$, and the number of insulin injections $m=3$.

Using the algorithm ALG1, we get the optimal control $c=[22.9582$ $-3.02735 .0941]$. Note that the second injection of insulin should be made with a negative amount, which is a nonsense from a medical point of view. The graphics of insulin shots and glucose dynamics are shown in Figure 1 and Figure 2, respectively.

Using ALG2 we remove the inconvenience of ALG1 and we obtain that the optimal doses of insulin are $c=\left[\begin{array}{lll}16.7446 & 0 & 4.0774\end{array}\right]$. In Figure 3 we can see the evolution of blood glucose concentration for this numerical experiment, with algorithm ALG2.

It is clear now that the second model (Eqn. (11)) works better than the first one (Eqn. (1)). The negative insulin amount obtained by ALG1 shows that the first model is not adapted for this example.

We intend to establish a treatment plan for a day. We have two possibilities: either we use ALG2 once for 12 hours and with the resulting final glucose concentration as initial concentration of glucose we apply again ALG2 for 12 hours, either we use ALG2 once for 24 hours (see Examples 2 to 4$)$. 
Example 2. We choose $L=12$ (hours), $G_{0}=2, A=1$, and the number of insulin injections $m=2$. Using ALG2, we get the optimal control $c=\left[\begin{array}{ll}13.5386 & 4.1833\end{array}\right]$. The final glucose concentration during 12 hours is $G_{\text {final }}=1.1562$.

Example 3. We take $L=12$ (hours), $G_{0}=1.1562$, which is the final glucose in the previous experiment, $A=1$, and the number of insulin injections $m=2$. With ALG2, we get the optimal control $c=\left[\begin{array}{ll}6.5897 & 5.8748\end{array}\right]$. We observe the stabilization of the amount of insulin injected.

Example 4. We now consider $L=24$ (hours), $G_{0}=2, A=1$, and the number of insulin injections $m=4$. Using ALG2 we obtain the following optimal control $c=\left[\begin{array}{llll}13.5305 & 4.44368 & 5.53 & 6.7421\end{array}\right]$.

The algorithm ALG1 works pretty well for the numerical data from Examples 2, 3 and 4.

Example 5. We consider the final time $L=18$ (hours), the initial concentration of glucose $G_{0}=2$, the desired level of glucose $A=0.9$, and the number of insulin injections $m=3$.

With ALG1 we obtain the optimal control $c=\left[\begin{array}{lll}18.9490 & 2.3965 & 5.9164\end{array}\right]$, and calculating the minimum, the mean and the final glucose concentration during 18 hours, we obtain $G_{\min }=0.7905, G_{\text {mean }}=0.9482, G_{\text {final }}=$ 1.0102 .

Using the second algorithm, ALG2, we get the optimal control

$c=\left[\begin{array}{lll}15.0738 & 3.5944 & 6.4703\end{array}\right]$. The minimum, the mean and the final glucose concentration during 18 hours are $G_{\min }=0.8037, G_{\text {mean }}=$ $0.92292, G_{\text {final }}=1.0127$. We observe that, the mean glucose is kept closer to the target value of glucose. In Figure 4 we can see the dynamics of the glucose concentration for this numerical experiment.

Let us also notice that in both examples the first insulin shot has a "big" value due to the initial glucose level $G(0)=G_{0}$ which is "far" from normal limits (the patient has hyperglicemia).

Example 6. We take the final time $L=18$ (hours), the initial concentration of glucose $G_{0}=1.0127$, which is the final glucose in the previous experiment, the desired level of glucose $A=0.9$, and the number of insulin injections $m=3$.

Using the second algorithm, ALG2 we get the optimal control $c=$ [6.1801 6.1135 5.9065]. The minimum, the mean, the maximum and the 
final glucose concentration during 18 hours are $G_{\min }=0.8324, G_{\text {mean }}=$ $0.8966, G_{\max }=1.0257, G_{\text {final }}=1.0239$. We observe that the mean glucose is kept closer to the target value of glucose and the bandwidth in which is varying the concentration of glucose is small. Taking into account the values of vector $c$ we notice the stabilization of insulin injections amount.

Examples 5 and 6 may be a treatment schedule for 36 hours for a patient with diabetes and initial hyperglicemia.

Example 7. We now consider the final time $L=36$ (hours), the initial concentration of glucose $G_{0}=2$, the desired level of glucose $A=0.9$, and the number of insulin injections $m=6$.

Using ALG2 we get the optimal control $c=\left[\begin{array}{lll}15.02866 & 3.7024 & 6.3348\end{array}\right.$ $6.01505 .90986 .0874]$. The mean glucose concentration during 36 hours are $G_{\text {mean }}=0.91$. The variation of glucose concentration for this numerical experiment is illustrated in the Figure 5.

We can compare the treatment resulting from Examples 5 and 6 to the one resulting from Example 7 .

We intend to configure a scheme of treatment for 2 days. We have two possibilities: either we use the algorithm ALG2 once for 24 hours and with the resulting final glucose concentration as initial concentration of glucose, we apply it again for 24 hours, either we use ALG2 once for 48 hours.

Example 8. We choose $L=24$ (hours), $G_{0}=2, A=0.9$, and the number of insulin injections $m=4$. Using ALG2, we get the optimal control $c=\left[\begin{array}{llll}15.0000 & 3.7692 & 6.2708 & 6.0280\end{array}\right]$. The final glucose concentration during 24 hours is $G_{\text {final }}=1.0181$.

We take next $L=24$ (hours), $G_{0}=1.0181, A=0.9$, and the number of insulin injections $m=4$. With ALG2, we get the optimal control $c=$ $\left[\begin{array}{llll}6.2599 & 6.0775 & 5.90 & 6.0885\end{array}\right]$. We observe the stabilization of the amount of insulin injected.

Example 9. We now consider $L=48$ (hours), $G_{0}=2, A=0.9$, and the number of insulin injections $m=8$. We obtain the follwing optimal control, using ALG2 $c=\left[\begin{array}{llllll}15.0284 & 3.6938 & 6.3697 & 5.9499 & 5.9766 & 6.0696\end{array}\right.$ $5.90146 .0892]$. In Figure 6 we can see the insulin shots for this numerical experiment.

The algorithm ALG1 works pretty well for the numerical data from Examples 8 and 9 . 
Here is another possibility to set up a treatment scheme for 2 days, with a smaller number of insulin injections (see Examples 10 to 12).

Example 10. We choose $L=24$ (hours), $G_{0}=2, A=0.9$, and the number of insulin injections $m=2$. Using ALG2, we get the optimal control $c=\left[\begin{array}{ll}16.9849 & 11.6044\end{array}\right]$. The final glucose concentration during 24 hours is $G_{\text {final }}=1.2676$.

Example 11. We consider $L=24$ (hours), $G_{0}=1.2676$, which is the final glucose in the previous experiment, $A=0.9$, and the number of insulin injections $m=2$. With ALG2, we obtain $c=\left[\begin{array}{ll}12.0279 & 12.0287\end{array}\right]$. We observe the stabilization of the amount of insulin injected.

Example 12. We now consider $L=48$ (hours), $G_{0}=2, A=0.9$, and the number of insulin injections $m=4$. We obtain the following optimal control, using ALG2 $c=\left[\begin{array}{llll}16.9969 & 11.6730 & 11.7632 & 12.2919\end{array}\right]$.

We can compare the treatment resulting from Examples 10 and 11 to the one resulting from Example 12.

The algorithm ALG1 works pretty well for the numerical data from Examples 10, 11 and 12.

The computing time for the program corresponding to ALG2 is of course much longer than the one for the program corresponding to ALG1 (an optimization problem vs. an algebraic linear system). As noticed the model given by Eqn. (11) is more sensitive than the one corresponding to Eqn. (1) for some experiments.

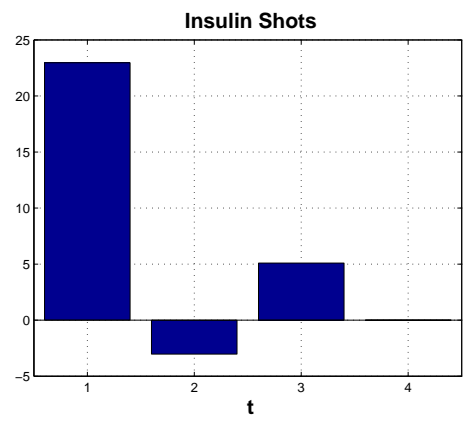

Figure 1. The insulin doses for 12 hours (ALG1)

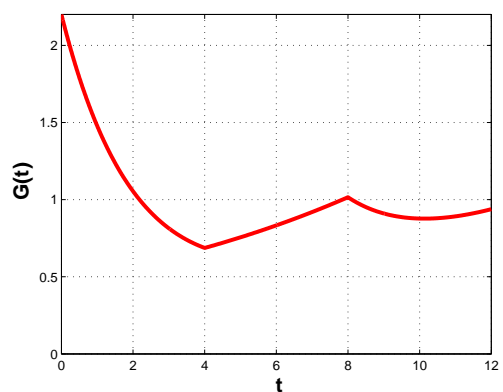

Figure 2. The glucose dynamics for Example 1(ALG1) 


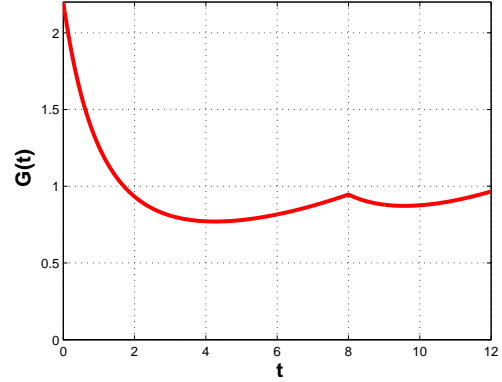

Figure 3. The glucose dynamics for Example 1 (ALG2)

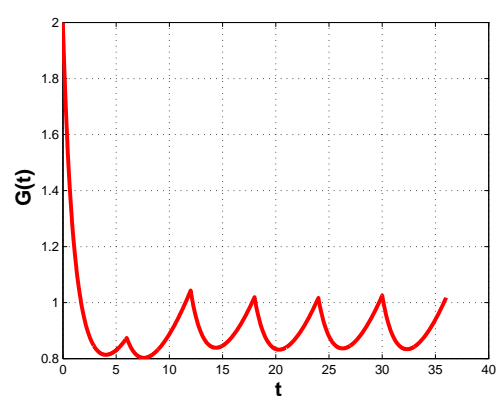

Figure 5. The glucose dynamics for Example 7 (ALG2)

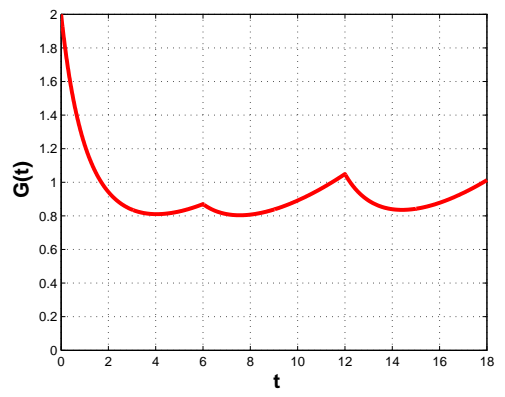

Figure 4. The glucose dynamics for Example 5 (ALG2)

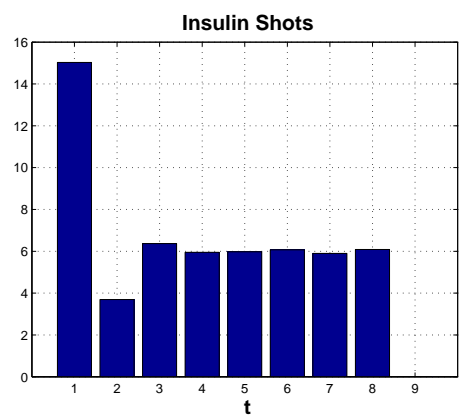

Figure 6. The insulin doses for 48 hours (ALG2)

\section{REFERENCES}

1. Ainseba, B.; AniţA, S. - Internal stabilizability for a reaction-diffusion problem modeling a predator-prey system, Nonlinear Anal., 61 (2005), 491-501.

2. AniţA, S.; AinsebA, B. - Internal eradicability for an epidemiological model with diffusion, Math. Biosci. Eng., 2 (2005), 437-443.

3. ArmiJo, L. - Minimization of functions having Lipschitz continuous first partial derivatives, Pacific J. Math., 16 (1966), 1-3. 
4. Arnăutu, V.; Neittannmäki, P. - Optimal Control from Theory to Computer Programs, Solid Mechanics and its Applications, 111, Kluwer Academic Publishers Group, Dordrecht, 2003.

5. Bolie, V.W. - Coefficients of blood glucose regulation, J. Applied Physiology, 16 (1961), 783-788.

6. Cherruault, Y. - Mathematical Modelling in Biomedicine. Optimal Control of Biomedical Systems, Mathematics and its Applications, 23, D. Reidel Publishing Co., Dordrecht, 1986.

7. De Gaetano, A.; Arino, O. - Mathematical modelling of the intravenous glucose tolerance test, J. Math. Biol., 40 (2000), 136-168.

8. Dura, G.; Moşneagu, A.-M. - Numerical approximation of Black-Scholes equation, An. Ştiinţ. Univ. "Al. I. Cuza" Iaşi. Mat., 56 (2010), 39-64.

9. FARIA, J.R. - Limit cycles in an optimal control problem of diabetes, Appl. Math. Lett., 16 (2003), 127-130.

10. Kalaba, R.; Spingarn, K. - Control, Identification, and Input Optimization, Mathematical Concepts and Methods in Science and Engineering, 25, Plenum Press, New York-London, 1982.

11. Keener, J.; Sneyd, J. - Mathematical Physiology, Interdisciplinary Applied Mathematics, 8, Springer-Verlag, New York, 1998.

12. Makroglou, A.; Li, J.; Kunng, Y. - Mathematical models and software tools for glucose-insulin regulatory system and diabetes: an overview, Appl. Numer. Math., 56 (2006), 559-573.

13. Parker, R.S.; Doyle III, F.J.; Peppas, N.A. - The intravenous route to bloode glucose control, IEEE Engrg. Medicine Biol., 20 (2001), 65-73.

14. Ruiz-Velázquez, E; Femat, R.; Campos-Delgado, D.U. - Blood glucose control for type I diabetes mellitus: A robust tracking $H_{\infty}$ problem, Control Engrg. Practice, 12 (2004), 1179-1195.

Received: 10.VI.2010

Faculty of Mathematics, "Al.I. Cuza" University of Iaşi, 700506, Iaşi, ROMANIA

varnautu@uaic.ro anamaria.mosneagu@uaic.ro 